Author manuscript

Heart. Author manuscript; available in PMC 2017 September 12.

Published in final edited form as:

Heart. 2017 July ; 103(14): 1089-1095. doi:10.1136/heartjnl-2016-310699.

\title{
Health literacy and warfarin therapy at two anticoagulation clinics in Brazil
}

Maria Auxiliadora Parreiras Martins ${ }^{1,2}$ Josiane Moreira Costa ${ }^{3}$ Juliana Vaz de Melo Mambrini $^{4}$, Antonio Luiz Pinho Ribeiro ${ }^{5}$, Emelia J Benjamin ${ }^{6,7,8}$, Luisa Campos Caldeira Brant $^{5}$, Michael K Paasche-Orlow ${ }^{9}$, and Jared W Magnani ${ }^{10}$

${ }^{1}$ Hospital das Clínicas and Faculdade de Farmácia, Universidade Federal de Minas Gerais, Belo Horizonte, Minas Gerais, Brazil

${ }^{2}$ Sections of Cardiovascular Medicine and Preventive Medicine, Boston Medical Center, Boston University School of Medicine, Boston, Massachusetts, USA

${ }^{3}$ Hospital Risoleta Tolentino Neves, Belo Horizonte, Minas Gerais, Brazil

${ }^{4}$ Centro de Pesquisa René Rachou/Fundação Oswaldo Cruz, Av. Augusto de Lima, Barro Preto, Belo Horizonte, Minas Gerais, Brazil

${ }^{5}$ Hospital das Clínicas and Faculdade de Medicina da Universidade Federal de Minas Gerais, Av. Prof. Alfredo Balena, Bairro Santa Efigênia, Belo Horizonte, Minas Gerais, Brazil

${ }^{6}$ Boston University and National Heart, Lung, and Blood Institute's Framingham Heart Study, Framingham, Massachusetts, USA

${ }^{7}$ Sections of Cardiovascular Medicine and Preventive Medicine, Boston Medical Center, Boston University School of Medicine, Boston, Massachusetts, USA

${ }^{8}$ Department of Epidemiology, Boston University School of Public Health, Boston, Massachusetts, USA

${ }^{9}$ General Internal Medicine, Boston University School of Medicine, Boston, Massachusetts, USA

${ }^{10}$ Department of Medicine, Division of Cardiology, UPMC Heart and Vascular Institute, University of Pittsburgh, Pittsburgh, Pennsylvania, USA

\begin{abstract}
Objective-Health literacy has been related to health-related conditions and health outcomes.

Studies examining the association of health literacy and anticoagulation have had variable results.
\end{abstract}

Correspondence to, Professor Maria Auxiliadora Parreiras Martins, Faculdade de Farmácia, Universidade Federal de Minas Gerais, Av. Antônio Carlos 6627, Campus Pampulha, Belo Horizonte, Minas Gerais, CEP 31270-901, Brazil;

auxiliadorapmartins@hotmail.com.

Contributors MAPM, ALPR, EJB, LCCB, MKPO and JWM have been directly involved in the conception of this manuscript, and interpreted the results in collaboration. MAPM and JMC collected data. JVMM assisted in performing statistical analysis. All authors have provided important contributions to drafting, editing and revising the manuscript. The authors take responsibility for all aspects of the reliability and freedom from bias of the data presented and its final contents.

Competing interests None declared.

Ethics approval Institutional Ethics Committee of the Universidade Federal de Minas Gerais

Provenance and peer review Not commissioned; externally peer reviewed. 
We sought to investigate the relations of health literacy and percentage of time in therapeutic range (TTR) in a vulnerable Brazilian cohort at two hospital-based anticoagulation clinics.

Methods-We measured health literacy with the Short Assessment of Health Literacy for Portuguese-speaking Adults (SAHLPA-18) in 2015-2016. We identified the demographic and clinical characteristics associated with health literacy and related health literacy to TTR.

Results-We enrolled 422 adults prescribed chronic warfarin therapy in our observational study (median age 62.1 years; $58.8 \%$ women; monthly income $\$ 200.00$ ). The prevalence of inadequate health literacy (score $0-14$ points) was $72.3 \%$ with a median score of 12 (quartiles, Q1=10; Q3=15) on the SAHLPA-18. The median TTR was $66.1 \%$. In the multivariable logistic analysis, cognitive impairment and assistance with taking warfarin were associated with inadequate health literacy. Prosthetic heart valves and more school years were associated with adequate health literacy. Our analyses showed no significant relation between health literacy and TTR, analysing health literacy as a categorical (adjusted OR 1.05 ; $95 \%$ CI 0.65 to 1.70 ) or continuous variable (Spearman's coefficient 0.02; $\mathrm{p}=0.70$ ).

Conclusions-Inadequate health literacy was highly prevalent in this impoverished Brazilian cohort receiving anticoagulation with warfarin. However, we did not identify an association between health literacy and TTR. Future investigations may consider the systemic factors that contribute towards successful anticoagulation outcomes for vulnerable patient cohorts with inadequate health literacy.

\section{INTRODUCTION}

Health literacy is established as a principal component of patient health. Health literacy has been related to severity of health-related conditions, prevention activities, process markers of health and control of chronic diseases, and health outcomes. ${ }^{1}$ The WHO has defined health literacy as 'the cognitive and social skills which determine the motivation and ability of individuals to gain access to, understand and use information in ways which promote and maintain good health. ${ }^{2}$ Inadequate health literacy has been reported to have a high prevalence across countries with estimates of $29 \%-62 \%$ in the USA and European countries. ${ }^{34}$ The prevalence of health literacy in lower income countries has not been precisely determined.

In some studies, adequate health literacy has been shown to contribute towards the successful use of oral anticoagulation. ${ }^{5-7}$ An association between health literacy and successful anticoagulation use is plausible because warfarin use is complicated by its narrow therapeutic index, wide variability in dose-response and need for frequent monitoring. ${ }^{8}$ Health literacy has been related to understanding the rationale for anticoagulation; however, investigation of the relationship between health literacy and successful warfarin use has yielded mixed results. ${ }^{569-11}$ Warfarin is the most widely used oral anticoagulant worldwide and studying health literacy in low-income and middle-income countries is relevant because of the increased vulnerability of such populations to health-related adversity and the likelihood of inadequate health literacy. Further, the most recent European Society of Cardiology guidelines have emphasised patient education and shared decision making as 
priorities for atrial fibrillation (AF) management and care. ${ }^{12}$ Evaluation of health literacy is critical towards engaging patients in shared decision making.

We employed the Short Assessment of Health Literacy for Portuguese-speaking Adults (SAHLPA-18), validated in Brazilian Portuguese by Apolinario et al, ${ }^{13}$ to examine health literacy in anticoagulation clinics in two large tertiary care settings. Health literacy has been referred to as a psychosocial risk factor for warfarin instability. ${ }^{14}$ The identification of sociodemographic and clinical factors associated with health literacy in individuals taking warfarin may guide interventions focused on tailored patient education strategies. We hypothesised that inadequate health literacy is related to poor anticoagulation control, as quantified by percentage of time in therapeutic range (TTR).

\section{METHODS}

\section{Study design and settings}

The present investigation is a cross-sectional, observational study conducted in the anticoagulation clinics of two public teaching hospitals in Belo Horizonte, Southeast Brazil. The hospitals are large tertiary and specialty care facilities that together treat approximately 35000 admissions and over 500000 ambulatory visits annually.

At both institutions, individuals prescribed warfarin are enrolled in multidisciplinary anticoagulation clinics with established protocols for patient education and warfarin dose adjustment. Participants in the current study were enrolled from July 2015 to February 2016. The study protocol was approved by the Institutional Ethics Committee of the Universidade Federal de Minas Gerais. All participants provided informed consent.

\section{Participants}

Study eligibility criteria were: age $\geq 18$ years, indication for warfarin therapy $\geq 60$ days continuously and $\geq 3$ international normalised ratio (INR) measures in the clinic. Individuals were assessed prospectively for eligibility at anticoagulation monitoring visits. Participants were excluded due to: inability to communicate secondary to dementia, aphasia or other cause; inability to communicate in Brazilian Portuguese; and anticoagulation clinic visit gaps of $\geq 90$ days. Participants who died within 90 days of enrolment were excluded from analyses.

\section{Health literacy measurement and variable assessment}

Health literacy was measured with SAHLPA-18, selected for its reliability in identifying low health literacy. ${ }^{15}$ It consists of 18 common medical terms that patients read aloud and associate with another word from a pair displayed by the interviewer. Score range is $0-18$ with $\leq 14$ indicating inadequate health literacy. ${ }^{13}$

Age and sex were extracted from the medical record. Race was self-reported and categorised as white or non-white. Education was obtained by self-report and categorised as none, incomplete elementary school, elementary school, incomplete high school or $\geq$ high school. Self-reported income was converted to US\$ using established exchange rates. Cohabitation was defined as living permanently with at least one person. 
Clinical data were collected from medical records, prescriptions, laboratory results and participant's self-report. Indication for warfarin therapy was categorised as AF, prosthetic heart valve, stroke/transient ischaemic attack and/or thromboembolism. Anticoagulation administration and INR goals were guided by standard practice guidelines. Duration in years of warfarin use was recorded as a continuous variable. Assistance with taking warfarin (yes/no) was determined as the consistent need of caregiver assistance for warfarin administration. Cognitive status was evaluated using a Brazilian version of the Mini-Mental State Examination (MMSE). ${ }^{16}$ MMSE scores were stratified by school years to categorise patients as impaired or normal cognitive function. ${ }^{17}$ Comorbidities are provided in online supplementary table $\mathrm{S} 1$. The number of comorbidities was calculated by summing all diagnoses, excluding the indication for warfarin therapy. The number of prescribed medications was obtained from medical records.

\section{Statistical analysis}

Categorical variables were described by their distributions and continuous measures as median and quartiles due to their non-normal distribution, combining data among hospitals. We quantified anticoagulation by TTR, measured to determine the percentage of time the INR was in the appropriate target range for each participant. ${ }^{18}$ The TTR was calculated using the Rosendaal method ${ }^{19}$ which entails a linear interpolation of INR values, expressed as a percentage, and requires the minimum number of two INR measurements to be done. INR values were obtained over the longest duration available from 2009 to 2015 . For participants who had INR intervals $>56$ days, we used INR values to calculate TTR for the valid intervals and then we used each separate TTR value to calculate the TTR.

We used logistic regression models to relate age, sex, race, school years, education, monthly income, cohabitation, indications for warfarin therapy, duration of warfarin use, assistance with taking warfarin, TTR, number of chronic medications, cognitive status, study site and number of comorbidities to inadequate health literacy. The selection of variables hypothesised to be associated with health literacy was based on previous publications. ${ }^{5611}$ Models were adjusted for age, sex and study site. Results were expressed as OR with $95 \%$ CIs. The variables associated with health literacy with $p$ value $<0.20$ were included in a multivariable logistic regression model using stepwise adjustments (method forward logistic regression) to identify sociodemographic and clinical characteristics related to health literacy.

To investigate the relations between health literacy and TTR, SAHLPA-18 scores were analysed in both a categorical and continuous manner. We considered health literacy as the independent variable and TTR as the dependent variable. We employed a multivariable logistic regression model using stepwise adjustments to select the covariates related to TTR that would remain in the model examining the relation between health literacy and TTR. We tested the following variables in the multivariable model: age, sex, study site, race, school years, income, cohabitation, presence of prosthetic heart valves, duration of warfarin use, assistance with taking warfarin, number of chronic medications, cognitive status, number of comorbidities and neuropsychiatric disorders. The Hosmer and Lemeshow test was used to verify the model fit ( $p>0.05$ ). A two-tailed $p$ value $<0.05$ was considered statistically 
significant. Consistent with prior analyses, we performed a continuous analysis of SAHLPA-18 score to identify a threshold effect in the relation of health literacy and TTR. ${ }^{20}$ We created five categories based on the examination of their frequency distribution, as previously done by Wolf et al. ${ }^{20}$ The categories were: $1=0-8$ points; $2=9-11$ points; $3=12$ 13 points; $4=14-15$ points; and 5=16-18 points. The linear correlation between health literacy and TTR was tested by the Spearman's coefficient with two-sided significance level of $5 \%$. A sensitivity analysis was performed to assess TTR using INR measures obtained during the most recent year of follow-up. Patients were classified as having poor TTR $(<60 \%)$ or adequate TTR $(\geq 60 \%) .^{21}$

Data entry was validated by double entry using EpiData software (V.3.1; EpiData Association, Odense M, Denmark). Data were analysed with the Statistical Package for Social Sciences (SPSS for Windows, V.21.0; SPSS, Chicago, Illinois, USA).

\section{RESULTS}

Of approximately 900 clinic participants, 540 were assessed for eligibility and 422 (78.1\%) were enrolled over a prospective, 8-month study duration. Figure 1 presents the study participant selection flow chart. Table 1 summarises the descriptive covariates of participants (median age 62.1 years; $58.8 \%$ women). There were 315 (74.6\%) participants with education <elementary school completion; median monthly income was approximately $\$ 200.00$. The main indications for warfarin use were AF $(n=314,74.4 \%)$ and prosthetic valves ( $\mathrm{n}=126,29.9 \%)$. The median duration of warfarin use was 2.7 years (Quartiles, $\mathrm{Q} 1=1.2 ; \mathrm{Q} 3=5.7)$ with a predominant INR target range of 2.0-3.0 $(\mathrm{n}=317 ; 75.1 \%)$. The median TTR was $66.1 \%(\mathrm{IQR}=18.7)$. We identified that $50.5 \%$ of participants had an INR interval $>56$ days; none had all INRs above this interval limit. There were 262 (62.1\%) patients with some degree of cognitive impairment. Participants had a high burden of chronic disease, as evidenced by the median number of comorbidities being $3(\mathrm{Q} 1=2$; Q3 = 5 ) and median number of chronic medications being $6(\mathrm{Q} 1=4$; Q3 = 7). Comorbidity distributions are described in online supplementary table S1.

\section{Measurements and factors associated to inadequate health literacy}

Most participants were classified as having inadequate health literacy (72.3\%) with a median score of 12 (Q1, 10; Q3, 15) on the SAHLPA-18. In models adjusted for age, sex and study site, inadequate health literacy was associated with fewer school years, prosthetic heart valves, duration of warfarin use, assistance with taking warfarin and cognitive status. For each additional 10 years of age, there was a $41 \%$ increased likelihood of inadequate health literacy (OR 1.41; 95\% CI 1.19 to 1.69). Each increase in year of education was associated with decreased likelihood of inadequate health literacy (OR $0.79 ; 95 \%$ CI 0.74 to 0.86 ). Table 2 presents the relation between decreasing health literacy and lower education.

Factors associated with inadequate health literacy in the multivariable analysis were cognitive impairment (OR 3.57; 95\% CI 2.05 to 6.23) and assistance with taking warfarin (OR 2.64; 95\% CI 1.02 to 6.84 ). Factors associated with adequate literacy in the multivariable analysis were having a prosthetic heart valve (OR 0.36 ; 95\% CI 0.20 to 0.64 ) 
and years of formal education (OR $0.76 ; 95 \%$ CI 0.69 to 0.82 ). The final model, presented in table 3 , had a good statistical fit using the Hosmer and Lemeshow test $(\mathrm{p}=0.34)$.

\section{Relation between health literacy and TTR}

The median TTR for inadequate health literacy and adequate health literacy was $65.4 \%$ $(56.4 ; 74.6)$ and $68.0 \%(56.2 ; 77.5)$, respectively. The association between TTR as a continuous measure and health literacy as the dependent variable was not significant in a logistic regression model adjusted for age, sex and study site (OR 1.01; 95\% CI 0.99 to 1.02). Likewise, we did not identify an association between health literacy and TTR in a logistic regression model adjusting for age, sex, study site, assistance with taking warfarin and number of chronic medications (OR 1.05 ; $95 \%$ CI 0.65 to 1.70 ; results summarised in table 4). This final model had a good statistical fit using the Hosmer and Lemeshow test $(p=0.08)$. The sensitivity analysis showed no significant difference in the estimates of health literacy and TTR using INR measures obtained during the most recent year of follow-up (online supplementary tables S2 and S3). Further, there was no consistent association in the relation of SAHLPA-18 as a categorical measure and TTR, when SAHLPA-18 was categorised into five discrete levels (figure 2). Our analysis showed no significant linear correlation between SAHLPA-18 scores and TTR (Spearman's coefficient 0.02; $p=0.70$ ). Hence, we were not able to identify a threshold effect for the relation of health literacy and TTR. Therefore, we sought to determine the effect size that we were powered to detect. We performed a post hoc determination of statistical power to assess a statistically significant difference in TTR between individuals with inadequate and adequate health literacy. We determined that our statistical power was limited (10.0\%) to identify such a difference given our sample size and the mean TTR for those with inadequate $(64.7 \% \pm 13.8 \%)$ and adequate $(65.8 \% \pm 15.5 \%)$ health literacy.

\section{DISCUSSION}

We identified a high prevalence of inadequate health literacy $(n=305,72.3 \%)$ in this lowincome patient cohort. However, the lack of relation of health literacy and TTR was an unexpected finding. In contrast to our hypothesis, we did not identify a significant relation between health literacy and TTR, analysing health literacy as a categorical or continuous variable. Previous studies assessing health literacy and anticoagulation outcomes have shown conflicting or unexpected results. ${ }^{5-71122}$ Several studies have identified significant associations of health literacy with TTR. ${ }^{5-7}$ In contrast, others have found no relation of health literacy and TTR in English-speaking and Spanish-speaking cohorts. ${ }^{1122}$ Of note, we found that the median TTR in our cohort was very high, even similar or greater than that observed in clinical trials (range $58.0 \%-68.4 \%$ ). ${ }^{23-25}$ It is possible that the absence of a spectrum of health literacy precluded identifying a relation between these variables, given that $>70 \%$ of the cohort had inadequate health literacy.

Following multivariable regression analysis, cognitive impairment and assistance with taking warfarin were associated with inadequate health literacy, whereas prosthetic heart valves and more years of formal education were associated with adequate health literacy. The association between cognitive impairment and inadequate health literacy is consistent with 
previous studies. ${ }^{2627}$ Cognitive assessments have been related to health literacy in chronic diseases such as heart failure ${ }^{26}$ or hypertension. ${ }^{27}$ As a related measure, individuals requiring assistance with taking warfarin had a 2.6-fold increased likelihood of having inadequate health literacy compared with those not requiring assistance. We expect that decreased health literacy would be concomitant with limited ability to self-manage medications and independently follow instructions.

Of interest, we identified that higher health literacy was associated with the implantation of a prosthetic heart valve as the indication for anticoagulation. We cannot exclude the possibility that biases such as socioeconomic resources, education or other unmeasured social determinants of health may influence selection for prosthetic heart valve surgery. Our finding that greater years of education were related to health literacy is consistent with prior studies. 52627

Our post hoc power calculations determined that we were underpowered to detect a difference in TTR by level of health literacy in our cohort. Our study consists of individuals enrolled in anticoagulation clinics linked to the Brazilian Public Health System in our region. More geographically and socioeconomically diverse populations may reveal different associations between health literacy and TTR.

Further, our study did not account for family support and social network that may influence warfarin adherence and in turn bolster TTR. Second, our study may have important selection biases. Individuals with inadequate health literacy may likely have had worse response to warfarin therapy and may have died prior to enrolment. Also, individuals with inadequate health literacy may have experienced barriers to healthcare access or have not been prescribed warfarin for a variety of medical and non-medical reasons. Our data suggest such biases because of the shorter duration of warfarin use in older individuals with inadequate health literacy compared with those with adequate health literacy.

Approximately one third of the Brazilian population presents low functional literacy and large regions of the country have even poorer literacy rates. ${ }^{28}$ Our study found that although there was high prevalence of inadequate health literacy among low-income people taking warfarin, the TTR was considerably high. From a clinical point of view, even people with inadequate health literacy may benefit from warfarin therapy if they have access to dedicated anticoagulation clinics. Our findings reinforce the need for considering health literacy when planning patient-centred educational interventions and delivering care to disadvantaged populations.

\section{Strengths and limitations}

The strengths of our study are that we assessed health literacy in a vulnerable patient population prospectively enrolled in tertiary healthcare centres. To our knowledge, this is the first study examining health literacy and metrics of anticoagulation in South America. Hence, the findings and investigation provide an important and novel benchmark for patientcentred investigations in health literacy and cardiovascular outcomes in Brazil. 
Our analysis has crucial limitations. First, this cohort was not intended to be representative of our clinical population, nor was it intended to include all patients receiving anticoagulation monitoring at these facilities. Participants were recruited sequentially. While we attempted to include as many clinic patients as possible, we do recognise the possibility of selection bias. Participants with greater comorbidities and disability, and potentially those with lower health literacy, may have been less likely to have used the clinic and had opportunities for enrolment. Likewise, we do not have information on individuals who were candidates for anticoagulation but not enrolled in our clinic, regardless of health literacy level. Second, the SALHPA-18 is not a comprehensive assessment of health literacy. Domains such as writing, listening, speaking, numeracy and conceptual knowledge ${ }^{29}$-not evaluated by the SALPHA-18 - may contribute towards health outcomes in individuals receiving anticoagulation. ${ }^{5}$ Third, we extracted information from medical records. Whereas this approach is consistent with many studies conducted in healthcare settings, we cannot exclude misclassification of comorbidity or diagnosis. Fourth, cut-off points for MMSE are heterogeneous in Brazilian instruments and validation still needs to be developed. Therefore, we may have misclassified cognitive status resulting in the high prevalence of cognitive impairment in our study. Fifth, our study was conducted in a single region of Brazil, and the generalisability of our study to higher socioeconomic cohorts, or across Brazilian states and regions or to other South American nations remains unknown. Finally, while we sought to include relevant sociodemographic and clinical measures, we expect there was residual confounding from variables, such as social determinants of health that were not collected in this investigation.

\section{CONCLUSIONS}

We determined that inadequate health literacy was highly prevalent in this socioeconomically impoverished Brazilian cohort of patients receiving anticoagulation. In contrast to our hypothesis, we did not identify an association between health literacy and TTR. High-quality anticoagulation clinics may contribute to overcome the identified disadvantages of inadequate health literacy. Future studies should identify systemic factors that facilitate successful anticoagulation outcomes for people with inadequate health literacy.

\section{Supplementary Material}

Refer to Web version on PubMed Central for supplementary material.

\section{Acknowledgments}

Funding MAPM is supported by the Conselho Nacional de Desenvolvimento Científico e Tecnológico- CNPq, Brazilian government programme for postdoctorate degree abroad (Grant No. 200639/2015-3). ALPR is a fellow of the Conselho Nacional de Desenvolvimento Científico e Tecnológico-CNPq. EJB is supported in part through NIH/NHLBI HHSN268201500001I; N01-HC25195, 2R01HL092577, 1R01HL128914. This award was supported by Grant 2015084 from the Doris Duke Charitable Foundation (JWM)

\section{References}

1. Dewalt DA, Berkman ND, Sheridan S, et al. Literacy and health outcomes: a systematic review of the literature. J Gen Intern Med. 2004; 19:1228-39. [PubMed: 15610334] 
2. World Health Organization. [accessed 13 Jun 2016] Health promotion glossary Geneva. 1988. http:// www.who.int/healthpromotion/about/HPR\%20Glossary\%201998.pdf

3. Sørensen K, Pelikan JM, Röthlin F, et al. HLS-EU Consortium. Health literacy in Europe: comparative results of the European health literacy survey (HLS-EU). Eur J Public Health. 2015; 25:1053-8. [PubMed: 25843827]

4. [accessed 23 Jun 2016] The health literacy of america's adults - Results from the 2003 national assessment of adult literacy. 2006. http://nces.ed.gov/pubs2006/2006483.pdf

5. Estrada CA, Martin-Hryniewicz M, Peek BT, et al. Literacy and numeracy skills and anticoagulation control. Am J Med Sci. 2004; 328:88-93. [PubMed: 15311167]

6. Oramasionwu CU, Bailey SC, Duffey KE, et al. The association of health literacy with time in therapeutic range for patients on warfarin therapy. J Health Commun. 2014; 19(Suppl 2):19-28. [PubMed: 25315581]

7. Lasater L, Davidson A, Mehler P, et al. Adherence and anticoagulation therapy outcomes: a preliminary report. J Gen Intern Med. 2003:179.

8. Ageno W, Gallus AS, Wittkowsky A, et al. Oral anticoagulant therapy: antithrombotic therapy and prevention of thrombosis, 9th ed: American college of chest physicians evidence-based clinical practice guidelines. 2012; 141:e44S-e88S.

9. Fang MC, Panguluri P, Machtinger EL, et al. Language, literacy, and characterization of stroke among patients taking warfarin for stroke prevention: implications for health communication. Patient Educ Couns. 2009; 75:403-10. [PubMed: 19171448]

10. Tang EO, Lai CS, Lee KK, et al. Relationship between patients' warfarin knowledge and anticoagulation control. Ann Pharmacother. 2003; 37:34-9. [PubMed: 12503930]

11. Fang MC, Machtinger EL, Wang F, et al. Health literacy and anticoagulation-related outcomes among patients taking warfarin. J Gen Intern Med. 2006; 21:841-6. [PubMed: 16881944]

12. Kirchhof P, Benussi S, Kotecha D, et al. ESC guidelines for the management of atrial fibrillation developed in collaboration with EACTS: the task force for the management of atrial fibrillation of the European Society of Cardiology (ESC). Eur Heart J. 2016; 2016:2893-962.

13. Apolinario D, Braga RC, Magaldi RM, et al. Short assessment of health literacy for Portuguesespeaking adults. Rev Saude Publica. 2012; 46:702-11. [PubMed: 22782124]

14. Diug B, Evans S, Lowthian J, et al. The unrecognized psychosocial factors contributing to bleeding risk in warfarin therapy. Stroke. 2011; 42:2866-71. [PubMed: 21836093]

15. Lee SY, Stucky BD, Lee JY, et al. Short assessment of health literacy—Spanish and English: a comparable test of health literacy for Spanish and English speakers. Health Serv Res. 2010; 45:1105-20. [PubMed: 20500222]

16. Brucki SM, Nitrini R, Caramelli $P$, et al. Suggestions for utilization of the mini-mental state examination in Brazil. Arq Neuropsiquiatr. 2003; 61:777-81. [PubMed: 14595482]

17. Crum RM, Anthony JC, Bassett SS, et al. Population-based norms for the Mini-Mental State Examination by age and educational level. JAMA. 1993; 269:2386-91. [PubMed: 8479064]

18. Veeger NJ, Piersma-Wichers M, Tijssen JG, et al. Individual time within target range in patients treated with vitamin $\mathrm{K}$ antagonists: main determinant of quality of anticoagulation and predictor of clinical outcome. A retrospective study of 2300 consecutive patients with venous thromboembolism. Br J Haematol. 2005; 128:513-9. [PubMed: 15686461]

19. Rosendaal FR, Cannegieter SC, van der Meer FJ, et al. A method to determine the optimal intensity of oral anticoagulant therapy. Thromb Haemost. 1993; 69:236-9. [PubMed: 8470047]

20. Wolf MS, Feinglass J, Thompson J, et al. In search of 'low health literacy': threshold vs. gradient effect of literacy on health status and mortality. Soc Sci Med. 2010; 70:1335-41. [PubMed: 20167411]

21. Connolly SJ, Pogue J, Eikelboom J, et al. ACTIVE W Investigators. Benefit of oral anticoagulant over antiplatelet therapy in atrial fibrillation depends on the quality of international normalized ratio control achieved by centers and countries as measured by time in therapeutic range. Circulation. 2008; 118:2029-37. [PubMed: 18955670]

22. Bryant B, Malone R, Ayscue D, et al. The effect of literacy and anticoagulation knowledge on the adequacy of warfarin anticoagulation for patients with atrial fibrillation. J Gen Intern Med. 2003:169. 
23. Wallentin L, Yusuf S, Ezekowitz MD, et al. RE-LY investigators. Efficacy and safety of dabigatran compared with warfarin at different levels of international normalised ratio control for stroke prevention in atrial fibrillation: an analysis of the RE-LY trial. Lancet. 2010; 376:975-83. [PubMed: 20801496]

24. Giugliano RP, Ruff CT, Braunwald E, et al. ENGAGE AF-TIMI 48 Investigators. Edoxaban versus warfarin in patients with atrial fibrillation. N Engl J Med. 2013; 369:2093-104. [PubMed: 24251359]

25. Patel MR, Mahaffey KW, Garg J, et al. ROCKET AF Investigators. Rivaroxaban versus warfarin in nonvalvular atrial fibrillation. N Engl J Med. 2011; 365:883-91. [PubMed: 21830957]

26. Morrow D, Clark D, Tu W, et al. Correlates of health literacy in patients with chronic heart failure. Gerontologist. 2006; 46:669-76. [PubMed: 17050758]

27. Levinthal BR, Morrow DG, Tu W, et al. Cognition and health literacy in patients with hypertension. J Gen Intern Med. 2008; 23:1172-6. [PubMed: 18459011]

28. Montenegro, IP. [accessed 13 Jun 2016] Indicadores de alfabetismo functional [Indicators for functional literacy]. 2006. http://www.ipm.org.br/pt-br/programas/inaf/Paginas/default.aspx

29. lynn Nielsen-Bohlman, AMP., Kindig, DA., editors. Health literacy: a prescription to end confusion. Washington: The National Academies Press; 2004. 


\section{Key messages}

What is already known on this subject?

- Health literacy has been related to severity of health-related conditions and health outcomes, especially to understanding the rationale for anticoagulation. Investigation results of the relationship between health literacy and successful warfarin use have been variable. Research on the relation of health literacy and anticoagulation in low-income and middleincome countries remains limited.

What might this study add?

- Our findings showed that inadequate health literacy was highly prevalent in a cohort of 422 impoverished Brazilians receiving anticoagulation with warfarin. In contrast to our hypothesis, there was no association between health literacy and percentage of time in therapeutic range.

How might this impact on clinical practice?

- Our results are relevant to clinical practice by showing that high-quality anticoagulation clinics may contribute to overcome the identified disadvantages of inadequate health literacy. Critical next steps are to improve warfarin-specific knowledge and numeracy skills in order to address challenges to health literacy that may result in adverse cardiovascular outcomes in individuals treated with anticoagulation. 
HRTN

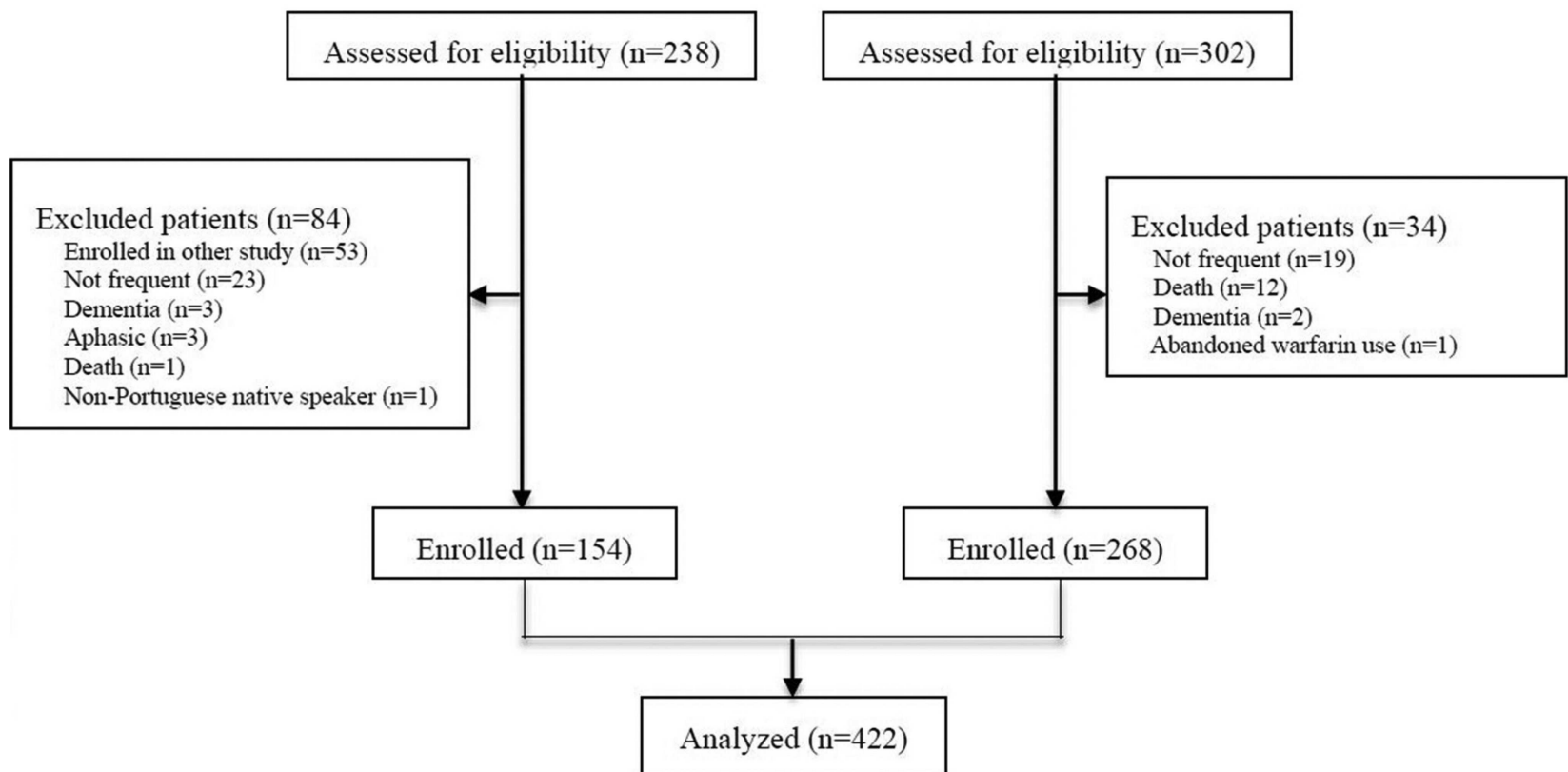

Figure 1.

Flow chart describing the selection of patients in anticoagulation clinics of two large public tertiary care centres in the Brazilian state of Minas Gerais. HC-UFMG, Hospital das Clinicas of the Universidade Federal de Minas Gerais; HRTN, Hospital Risoleta Tolentino Neves. 


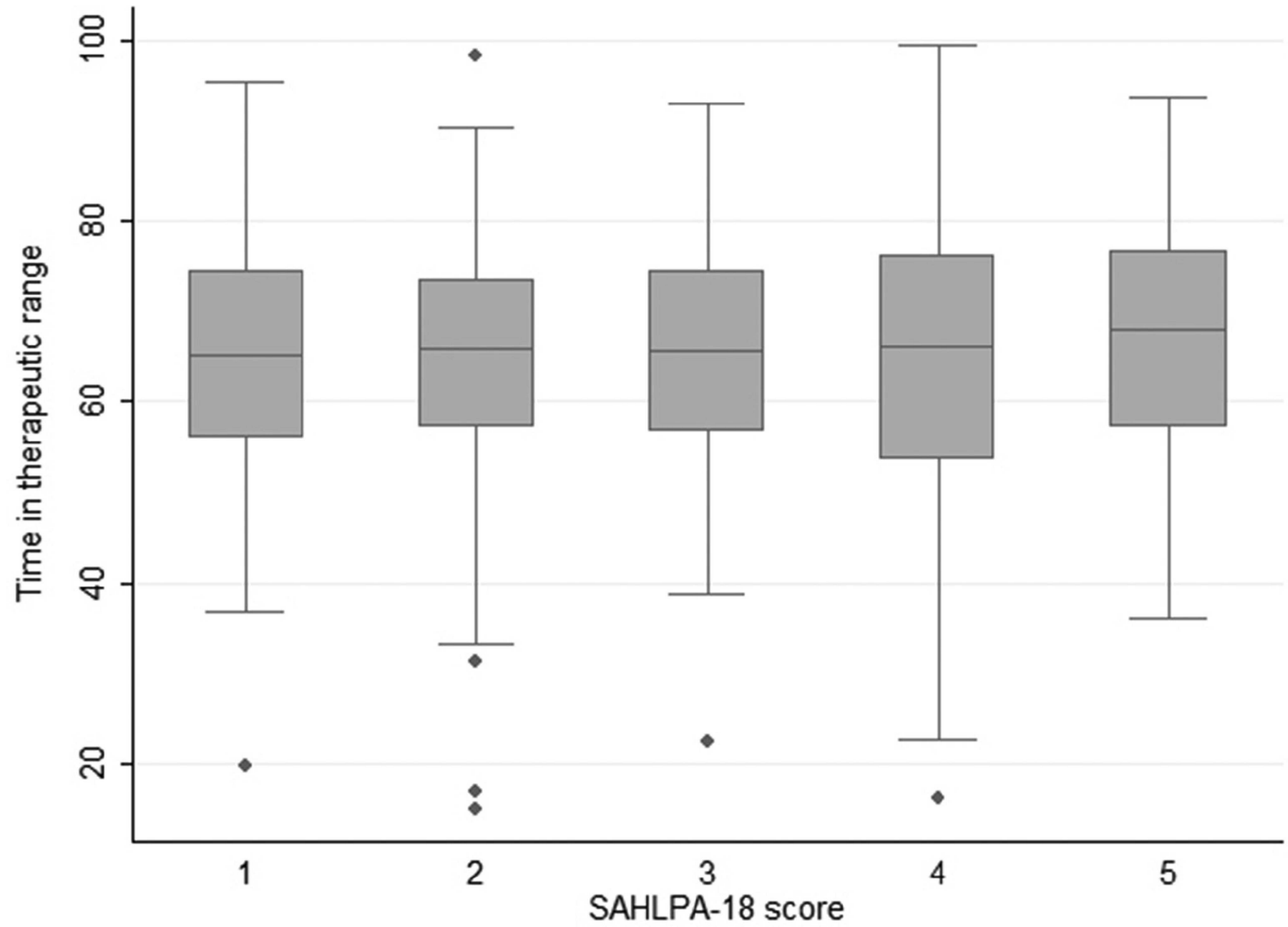

Figure 2.

Differences in time in therapeutic range according to distributions of SALHPA-18 score. The categories for SAHLPA- 18 score, based on frequency distributions, were: $1=0-8$ points $(\mathrm{n}=74) ; 2=9-11$ points $(\mathrm{n}=94) ; 3=12-13$ points $(\mathrm{n}=90) ; 4=14-15$ points $(\mathrm{n}=83)$; and $5=16-$ 18 points $(\mathrm{n}=81)$. SAHLPA-18, Short Assessment of Health Literacy for Portuguesespeaking Adults. 
Table 1

Descriptive data of study participants by hospital $(n=422)$

\begin{tabular}{|c|c|c|c|}
\hline Characteristics & Total $(n=422)$ & $\operatorname{HRTN}(n=154)$ & HC-UFMG $(n=268)$ \\
\hline Age, median (Q1; Q3) & $62.1(53.9 ; 71.8)$ & $64.8(56.8 ; 74.4)$ & $60.8(51.2 ; 69.6)$ \\
\hline \multicolumn{4}{|l|}{ Sex, n (\%) } \\
\hline Female & $248(58.8)$ & $88(57.1)$ & $160(59.7)$ \\
\hline Male & $174(41.2)$ & $66(42.9)$ & $108(40.3)$ \\
\hline \multicolumn{4}{|l|}{ Race, n (\%) } \\
\hline Non-white & $293(69.4)$ & $116(75.3)$ & $177(66.0)$ \\
\hline White & $129(30.6)$ & $38(24.7)$ & $91(34.0)$ \\
\hline School years, median (Q1; Q3) & $5.0(4.0 ; 8.0)$ & $5.0(5.0 ; 9.0)$ & $4.0(3.0 ; 7.0)$ \\
\hline \multicolumn{4}{|l|}{ Education, n (\%) } \\
\hline No education & $44(10.4)$ & $21(13.6)$ & $23(8.6)$ \\
\hline Incomplete elementary school & $271(64.2)$ & $89(57.8)$ & $182(67.9)$ \\
\hline Elementary school & $42(10.0)$ & $20(13.0)$ & $22(8.2)$ \\
\hline Incomplete high school & $13(3.1)$ & $7(4.6)$ & $6(2.2)$ \\
\hline$\geq$ High school & $52(12.3)$ & $17(11.0)$ & $35(13.1)$ \\
\hline Monthly income (US\$ ${ }^{*}$, median (Q1; Q3) & $200.6(100.3 ; 200.6)$ & $200.6(200.6 ; 225.1)$ & $167.13(100.3 ; 200.6)$ \\
\hline \multicolumn{4}{|l|}{ Cohabitation, $\mathrm{n}(\%)$} \\
\hline Yes & $374(88.6)$ & $133(86.4)$ & $241(89.9)$ \\
\hline No & $48(11.4)$ & $21(13.6)$ & $27(10.1)$ \\
\hline \multicolumn{4}{|l|}{ Indication for warfarin therapy, $\mathrm{n}(\%)^{\dagger}$} \\
\hline AF/Flutter & $314(74.4)$ & $122(79.2)$ & $192(71.6)$ \\
\hline Prosthetic heart valves & $126(29.9)$ & $17(11.0)$ & $109(40.7)$ \\
\hline Stroke/transient ischaemic attack & $72(17.1)$ & $57(37.0)$ & $15(5.6)$ \\
\hline Thromboembolism & $9(2.1)$ & $2(1.3)$ & $7(2.6)$ \\
\hline Duration of warfarin use (years), median (Q1; Q3) & $2.7(1.2 ; 5.7)$ & $2.1(1.2 ; 3.5)$ & $3.9(1.3 ; 6.5)$ \\
\hline \multicolumn{4}{|l|}{ Assistance with taking warfarin, $\mathrm{n}(\%)$} \\
\hline Yes & $56(13.3)$ & $29(18.8)$ & $27(10.1)$ \\
\hline No & $366(86.7)$ & $125(81.2)$ & $241(89.9)$ \\
\hline TTR $(\%$,$) median (Q1; Q3)$ & $66.1(56.2 ; 75.0)$ & $67.8(57.0 ; 76.7)$ & $65.4(56.0 ; 73.6)$ \\
\hline SAHLPA-18 score, median (Q1; Q3) & $12(10 ; 15)$ & $12(9 ; 14)$ & $13(10 ; 15)$ \\
\hline \multicolumn{4}{|l|}{ Health literacy level $\not{t}, \mathrm{n}(\%)$} \\
\hline Inadequate & $305(72.3)$ & $116(75.3)$ & $189(70.5)$ \\
\hline Adequate & $117(27.7)$ & $38(24.7)$ & $79(29.5)$ \\
\hline
\end{tabular}




\begin{tabular}{|c|c|c|c|}
\hline Characteristics & Total $(n=422)$ & HRTN (n=154) & HC-UFMG $(n=268)$ \\
\hline \multicolumn{4}{|l|}{ Categories of SAHLPA-18 score, $\mathrm{n}(\%)$} \\
\hline $0-8$ & $74(17.5)$ & $35(22.7)$ & $39(14.6)$ \\
\hline $9-11$ & $94(22.3)$ & $39(25.3)$ & $55(20.5)$ \\
\hline $12-13$ & $90(21.3)$ & $23(14.9)$ & $67(25.0)$ \\
\hline $14-15$ & $83(19.7)$ & $32(20.9)$ & $51(19.0)$ \\
\hline $16-18$ & $81(19.2)$ & $25(16.2)$ & $56(20.9)$ \\
\hline Number of chronic medications, median (Q1; Q3) & $6(4 ; 7)$ & $6(5 ; 7)$ & $6(4 ; 7)$ \\
\hline \multicolumn{4}{|l|}{ Cognitive status, $\mathrm{n}(\%)$} \\
\hline Impairment & $262(62.1)$ & $128(83.1)$ & $134(50.0)$ \\
\hline Normal function & $160(37.9)$ & $26(16.9)$ & $134(50.0)$ \\
\hline Number of comorbidities $\mathcal{\xi}$, median (Q1; Q3) & $3(2 ; 5)$ & $5(4 ; 6)$ & $2(1 ; 4)$ \\
\hline \multicolumn{4}{|c|}{ * Currency conversion, US $\$ 1.00=3.61$ Brazilian Reais (3/30/2016). } \\
\hline \multicolumn{4}{|c|}{ Total percentage is higher than $100 \%$ because a patient could have more than one indication for warfarin therapy. } \\
\hline \multicolumn{4}{|c|}{ SAHLPA-18 score, inadequate health literacy $=0-14$ points, adequate health literacy $=15-18$ points. } \\
\hline
\end{tabular}

AF, atrial fibrillation; HC-UFMG, Hospital das Clínicas of the Universidade Federal de Minas Gerais; HRTN, Hospital Risoleta Tolentino Neves; Q1, quartile 1; Q3, quartile 3; SAHLPA-18, Short Assessment of Health Literacy for Portuguese-speaking Adults; TTR, time in therapeutic range. 
Table 2

Statistical analysis adjusted for age, sex and study site with covariates of study participants by health literacy $(n=422)$

\begin{tabular}{|c|c|c|c|c|}
\hline Covariates & $\begin{array}{l}\text { Inadequate health } \\
\text { literacy }^{*}(n=305)\end{array}$ & $\begin{array}{l}\text { Adequate health } \\
\text { literacy }^{*}(n=117)\end{array}$ & $\begin{array}{l}\text { Adjusted OR of inadequate } \\
\text { health literacy (95\% CI) }\end{array}$ & $\mathbf{p}$ \\
\hline Age, median (Q1; Q3) & $63.5(55.3 ; 73.5)$ & $58.7(50.1 ; 65.9)$ & $1.41(1.19 \text { to } 1.69)^{\dagger}$ & $<0.001$ \\
\hline \multicolumn{5}{|l|}{ Sex, n $(\%)$} \\
\hline Male & $129(42.3)$ & $45(38.5)$ & $1.11(0.71$ to 1.74$)$ & 0.64 \\
\hline Female & $176(57.7)$ & $72(61.5)$ & Referent & \\
\hline \multicolumn{5}{|l|}{ Race, n (\%) } \\
\hline White & $96(31.5)$ & $33(28.2)$ & $1.13(0.70$ to 1.83$)$ & 0.62 \\
\hline Non-white & $209(68.5)$ & $84(71.8)$ & Referent & \\
\hline School years, median (Q1; Q3) & $5.0(3.0 ; 5.0)$ & $6.0(4.0 ; 11.0)$ & $0.79(0.74 \text { to } 0.86)^{\%}$ & $<0.001$ \\
\hline \multicolumn{5}{|l|}{ Education, n (\%) } \\
\hline No education & $42(13.8)$ & $2(1.7)$ & Referent & \\
\hline Incomplete elementary school & $208(68.2)$ & $63(53.9)$ & $0.18(0.04$ to 0.79$)$ & 0.02 \\
\hline Elementary school & $25(8.2)$ & $17(14.5)$ & $0.09(0.02$ to 0.43$)$ & $<0.01$ \\
\hline Incomplete high school & $7(2.3)$ & $6(5.1)$ & $0.07(0.01$ to 0.45$)$ & $<0.01$ \\
\hline$\geq$ High school & $23(7.5)$ & $29(24.8)$ & $0.05(0.01$ to 0.23$)$ & $<0.001$ \\
\hline Monthly income (US\$ $\mathcal{\xi}$ ), median (Q1; Q3) & $201(100 ; 201)$ & $201(127 ; 263)$ & $0.99(0.99$ to 1.00$)$ & $<0.01$ \\
\hline \multicolumn{5}{|l|}{ Cohabitation, n (\%) } \\
\hline Yes & $268(87.9)$ & $106(90.6)$ & $0.90(0.43$ to 1.86$)$ & 0.77 \\
\hline No & $37(12.1)$ & $11(9.4)$ & Referent & \\
\hline \multicolumn{5}{|l|}{ Indication for warfarin therapy, $\mathrm{n}(\%)$} \\
\hline \multicolumn{5}{|l|}{$\mathrm{AF} /$ flutter } \\
\hline Yes & $230(75.4)$ & $84(71.8)$ & $0.86(0.51$ to 1.44$)$ & 0.56 \\
\hline No & $75(24.6)$ & $33(28.2)$ & Referent & \\
\hline \multicolumn{5}{|l|}{ Prosthetic heart valves } \\
\hline Yes & $72(23.6)$ & $54(46.1)$ & $0.45(0.27$ to 0.76$)$ & $<0.01$ \\
\hline No & $233(76.4)$ & $63(53.9)$ & Referent & \\
\hline \multicolumn{5}{|l|}{ Stroke/transient ischaemic attack } \\
\hline Yes & $57(18.7)$ & $15(12.8)$ & $1.44(0.74$ to 2.85$)$ & 0.29 \\
\hline No & $248(81.3)$ & $102(87.2)$ & Referent & \\
\hline \multicolumn{5}{|l|}{ Thromboembolism } \\
\hline Yes & $7(2.3)$ & $2(1.7)$ & $1.60(0.32$ to 8.07$)$ & 0.57 \\
\hline No & $298(97.7)$ & $115(98.3)$ & Referent & \\
\hline
\end{tabular}




\begin{tabular}{|c|c|c|c|c|}
\hline Covariates & $\begin{array}{l}\text { Inadequate health } \\
\text { literacy* }_{(n=305)}\end{array}$ & $\begin{array}{l}\text { Adequate health } \\
\text { literacy }^{*}(n=117)\end{array}$ & $\begin{array}{l}\text { Adjusted OR of inadequate } \\
\text { health literacy }(95 \% \mathrm{CI})\end{array}$ & $\mathbf{p}$ \\
\hline Duration of warfarin use (years), median (Q1; Q3) & $2.5(1.1 ; 5.1)$ & $4.1(1.4 ; 6.7)$ & $0.93(0.88$ to 0.99$)$ & 0.02 \\
\hline \multicolumn{5}{|l|}{ Assistance with taking warfarin, $\mathrm{n}(\%)$} \\
\hline Yes & $255(83.6)$ & $111(94.9)$ & $3.16(1.30$ to 7.72$)$ & 0.01 \\
\hline No & $50(16.4)$ & $6(5.1)$ & Referent & \\
\hline TTR (\%), median (Q1; Q3) & $65.4(56.4 ; 74.6)$ & $68.0(56.2 ; 77.5)$ & $1.01(0.99$ to 1.02$)$ & 0.40 \\
\hline Number of chronic medications, median (Q1; Q3) & $6(4 ; 7)$ & $6(5 ; 7)$ & $0.92(0.83$ to 1.01$)$ & 0.09 \\
\hline \multicolumn{5}{|l|}{ Cognitive status, $\mathrm{n}(\%)$} \\
\hline Impairment & $206(67.5)$ & $56(47.9)$ & 2.54 (1.57 to 4.10$)$ & $<0.001$ \\
\hline Normal function & $99(32.5)$ & $61(52.1)$ & Referent & \\
\hline Number of comorbidities, median`(Q1; Q3) & $3(2 ; 5)$ & $3(2 ; 4)$ & $0.96(0.84$ to 1.10$)$ & 0.56 \\
\hline
\end{tabular}

'Per 10 years of age.

tPer year of educational attainment.

$\xi_{\text {Currency conversion, US } \$ 1.00=\mathrm{R} \$ 3.61 \text { Brazilian Reais }(3 / 30 / 2016)}$

9/The list of comorbidities included: Chagas disease, coronary artery disease, diabetes, dyslipidemia, gastrointestinal disease, chronic heart failure, haematological disease, hypertension, hypothyroidism, kidney dysfunction, liver failure, morbid obesity, neoplasia, neuropsychiatric disease, osteoarticular disease, peripheral vascular disease, respiratory disease, rheumatic disease and valve disease.

$\mathrm{AF}$, atrial fibrillation; $\mathrm{Q} 1$, quartile 1; Q3, quartile 3; TTR, time in therapeutic range 


\section{Table 3}

Logistic regression model for inadequate health literacy in 422 participants from two anticoagulation clinics *

\begin{tabular}{lll}
\hline Variables & OR $(\mathbf{9 5} \% \mathbf{C I})$ & $\mathbf{p}$ \\
\hline Age $^{\dagger}$ & $1.00(0.81$ to 1.24$)$ & 0.99 \\
\hline Sex & & \\
Male & $1.35(0.81$ to 2.25$)$ & 0.25 \\
Female & Referent & \\
\hline Study site & & \\
HRTN & $0.77(0.43$ to 1.40$)$ & 0.40 \\
HC-UFMG & Referent & \\
\hline Cognitive status & & \\
Impairment & $3.57(2.05$ to 6.23$)$ & $<0.001$ \\
Normal function & Referent & \\
\hline Assistance with taking warfarin & & $<0.001$ \\
Yes & $2.64(1.02$ to 6.84$)$ & 0.046 \\
No & Referent & \\
\hline Prosthetic heart valves & & \\
Yes & $0.36(0.20$ to 0.64$)$ & $<0.001$ \\
\hline No & Referent & \\
\hline School years ${ }^{*}$ & & \\
\hline
\end{tabular}

* The variables age, sex and study site were forced into the final stepwise model.

골

'Per 10 years of age.

Per year of educational attainment

HC-UFMG, Hospital das Clinicas of the Universidade Federal de Minas Gerais; HRTN, Hospital Risoleta Tolentino Neves. 
Table 4

Health literacy and TTR $(n=422)$

\begin{tabular}{lllll}
\hline Outcome & Health literacy $^{*}$ & $\mathbf{n}(\mathbf{( \% )}$ & OR $^{\prime}(\mathbf{9 5 \%} \mathbf{C I})$ & $\mathbf{p}$ \\
\hline $\mathrm{TTR}<60 \%, \mathrm{n}=147$ & Inadequate & $108(35.4)$ & $1.05(0.65$ to 1.70$)$ & 0.84 \\
& Adequate & $39(33.3)$ & Referent & \\
\hline
\end{tabular}

SAHLPA-18 score, inadequate health literacy $=0-14$ points, adequate health literacy $=15-18$ points.

${ }^{\dagger}$ Stepwise logistic regression model adjusted for age, sex, study site, assistance with taking warfarin and number of chronic medications.

TTR, time in therapeutic range.

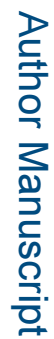

를

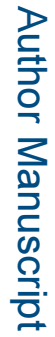

